\title{
The AMIGA Muon Counters of the Pierre Auger Observatory: Performance and Studies of the Lateral Distribution Function
}

\author{
Brian Wundheiler ${ }^{* a}$ for the Pierre Auger Collaboration ${ }^{b}$ \\ ${ }^{a}$ Instituto de Tecnologías en Detección y Astropartículas (CNEA, CONICET, UNSAM), \\ Av. Gral. Paz 1499 (1650) Buenos Aires, Argentina. \\ ${ }^{b}$ Observatorio Pierre Auger, Av. San Martín Norte 304, 5613 Malargüe, Argentina. \\ E-mail: auger_spokespersons@fnal.gov \\ Full author list: http://www.auger.org/archive/authors_2015_06.html
}

The AMIGA enhancement (Auger Muons and Infill for the Ground Array) of the Pierre Auger Observatory consists of a $23.5 \mathrm{~km}^{2}$ infill area where air shower particles are sampled by waterCherenkov detectors at the surface and by $30 \mathrm{~m}^{2}$ scintillation counters buried $2.3 \mathrm{~m}$ underground. The Engineering Array of AMIGA, completed since February 2015, includes 37 scintillator modules $\left(290 \mathrm{~m}^{2}\right)$ in a hexagonal layout. In this work, the muon counting performance of the scintillation detectors is analysed over the first 20 months of operation. Parametrisations of the detector counting resolution and the lateral trigger probability are presented. Finally, preliminary results on the observed muon lateral distribution function (LDF) are discussed.

The 34th International Cosmic Ray Conference,

30 July- 6 August, 2015

The Hague, The Netherlands

\footnotetext{
*Speaker.
} 


\section{Introduction}

The Pierre Auger Observatory [1], located in the province of Mendoza in Argentina, is a hybrid detector covering $3000 \mathrm{~km}^{2}$ with 1660 surface stations (the surface detector, SD) and 27 fluorescence telescopes (the Fluorescence Detector, FD). The SD stations are separated by $1.5 \mathrm{~km}$, while the telescopes are split in four buildings at the edge of the surface array, and point towards the atmosphere and the centre of the array. Currently, the Auger Observatory is being upgraded and AMIGA is one of the enhancement projects [2].

AMIGA consists of 61 detector pairs, each composed of a surface water-Cherenkov detector (SD infill) and a buried $30 \mathrm{~m}^{2}$ Muon Counter (MC). The AMIGA MCs are arranged on a $750 \mathrm{~m}$ triangular grid to directly measure the muon content of showers with primary energies $\geq 3 \times 10^{17} \mathrm{eV}$. The complete AMIGA array will cover an area of $23.5 \mathrm{~km}^{2}$ providing sufficient statistics given the higher rate of the sub-EeV showers. Important results on cosmic ray physics by means of muon detection techniques have been obtained previously by the Haverah Park [3], Akeno [4], Yakutsk [5] and AGASA experiments [6] and more recently by the KASCADE [7] and KASCADE-Grande [8] experiments.

The Engineering Array of AMIGA, called the Unitary Cell (UC), is a hexagon in the Observatory infill area (750 m spacing) with $2.3 \mathrm{~m}$-deep buried MCs at each hexagon vertex and at its centre (see Fig. 1). It has been completed since February 2015. Each of these 7 MCs is composed of 4 scintillation modules $(\mathrm{SM})$, of either $2 \times 5 \mathrm{~m}^{2}$ or $2 \times 10 \mathrm{~m}^{2}$ area, comprising 64 plastic scintillation bars sealed in a PVC casing, containing wavelength-shifting optical fibres, a 64 multianode photomultiplier tube, and acquisition electronics. The light produced in these bars is collected and propagated along the fibres which couple to the multi-pixel PMTs. Segmentation was selected since it permits the AMIGA counter electronics to just count pulses above a given threshold (see Sec. 3), without a detailed study of signal structure or peak amplitude/charge. In two UC positions, twin detectors are deployed consisting of four extra SMs at the KT position identified in Fig. 1 and three $10 \mathrm{~m}^{2}$ extra ones at the PC position. At the latter, there are also two extra $10 \mathrm{~m}^{2} \mathrm{SMs}$ deployed at $1.3 \mathrm{~m}$ depth. There is a SM with 4 SiPMs installed so that in total there are 37 deployed and working SMs.

In this work we analyse the data set from the first two years of UC operation. We evaluate the response of the twin position at KT to assess the muon counting

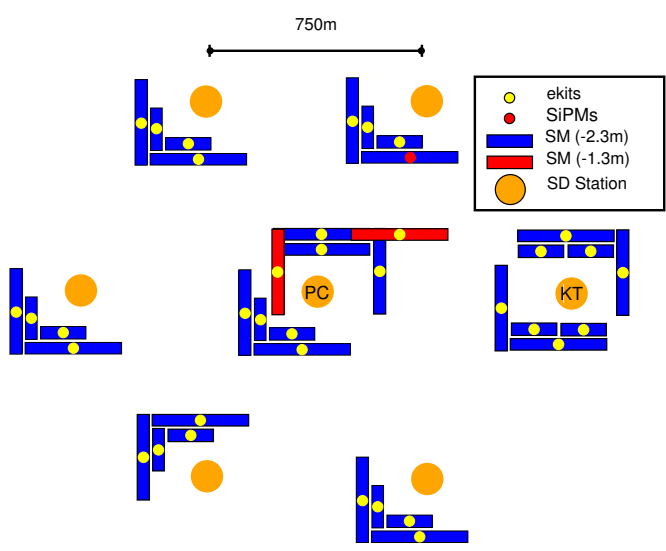

Figure 1: AMIGA Unitary Cell as of February 2015. The $5 \mathrm{~m}^{2}$ detectors at the KT and PC positions have been used for this analysis. resolution of the MCs, and get a parametrisation that allows us to establish the counting uncertainty of single events. We find the scaling between the muon number at a reference distance from the shower core and the energy 
of the primary cosmic ray, and we study the lateral distribution function (LDF) of the observed muons and obtain an effective value for its slope. This value can be used for single event fitting, leaving only the relevant composition-sensitive observable of the LDF, i.e. the number of muons at an optimal distance from the shower axis, to be determined from the fit.

\section{Design Considerations}

Having described the scintillator modules it is worth briefly describing the path followed by the light travelling through the optical fibre until the signal is processed by the Central Data Acquisition System (CDAS) at the Observatory campus. The 64 optical fibres are matched to a 64-pixel PMT through a custom-tailored optical alignment device. The PMT chosen is the Hamamatsu ultra bialkaline H8804-200MOD, a H7546 type PMT but with a different casing and an increased quantum efficiency peaking around $350 \mathrm{~nm}$.

The electronics of the MCs is split into two components, the underground electronics installed in each buried module and a reduced electronics at the surface. Both are powered by solar panels. The underground electronics includes the PMT, the front-end, digital, slow control, and power distribution boards and a data transmission unit. The surface electronics comprises an interface with the SD electronics (to get the trigger from the SD and to transfer muon data), the wireless communication to CDAS, the network switch, and the power regulator. The analogue front-end holds the pre-amplifiers and discriminators which are remotely set to an adjustable fraction of the average Single Photo Electron (SPE) amplitude of each PMT pixel. Thus, PMT pulses are converted into a train of digital 0's and 1's corresponding to the presence or absence of a signal above the aforementioned threshold. One bit per channel is saved in the front-end memory forming a 64-bit string. This conversion is performed in $3.125 \mathrm{~ns}$ time bins by a field-programmable gate array (FPGA). The memory consists of two circular buffers that store 2048 bins of 64 bits. These bit trains are stored following an SD trigger, recovered and transmitted upon a request from the CDAS. The MC event acquisition is synchronised at the lowest (hardware) level to the surface stations through a dedicated triggering line. The MC electronics maintains synchronisation, through a time-tagging scheme, mostly implemented in the FPGA. An event data trigger request, received by the surface radio, is sent from the surface to the underground microcontroller through an Ethernet line. The FPGA searches for the requested event and retrieves data. A counting strategy searches offline for the muon traces by inspecting the individual SPE signatures. As the vast majority of contaminating events produce only a sole SPE, by requiring at least a $1 \mathrm{X} 1$ string (with X either a 1 or a 0 ) for a muon footprint, accidentals are removed. Consequently, most of the accidental data, such as crosstalk or thermal photoelectrons are discarded. Muons are counted in time windows of $25 \mathrm{~ns}$, the duration corresponding to the detector dead time given by the width of the muon pulse due to the scintillator and fibre decay times (for details on counting techniques and performance see [9]).

\section{Calibration Routine and Data Set}

Due to the one-bit electronics technique, the discriminator levels, set relative to the mean pixel SPE amplitude, play a major role. Setting them too high will cause a loss of counting efficiency 

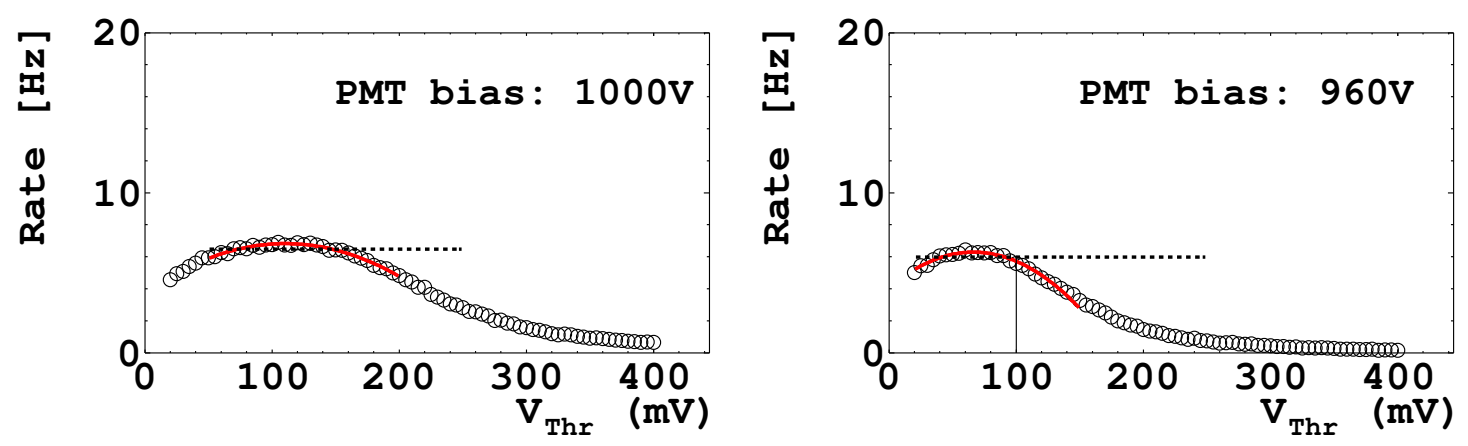

Figure 2: Background-radiation rates as a function of threshold, measured at KT for a $5 \mathrm{~m}^{2}$ module. Also shown are fits to the plateau regions in red (dotted line at $5 \%$ below the maxima). (Left) PMTs were biased at $1000 \mathrm{~V}$ as of March 2015. (Right) PMTs were biased at $960 \mathrm{~V}$ in the previous period, the full line showing the $100 \mathrm{mV}$ threshold fixed for all channels in the data set analysed in this work.

since the digital output of the discriminator might be too short for the $3.125 \mathrm{~ns}$ sampling period. Too low thresholds might produce two or three adjacent positive digital samples (i.e. 1's) for a SPE. The aim of the one-bit background-radiation calibration is to identify a feature (e.g. a plateau) in the behaviour of the rates as a function of the SPE threshold. Setting each individual threshold on its plateau will render a fully efficient detection system. The calibration induces the MCs to self-trigger which is not performed in the usual air-shower detection mode when the counters make use of their accompanying SD trigger.

In Fig. 2 (left panel) we show a typical distribution of the background-radiation rate from a single channel of a $5 \mathrm{~m}^{2}$ module measured with its PMT biased at $1000 \mathrm{~V}$. Each PMT was characterised prior to deployment with a mounted opto-electronics device. A plateau-like structure is clearly apparent within the range from $80-190 \mathrm{mV}$ (i.e. $25 \%$ to $45 \%$ of the SPE). In stable operational mode, the pixel calibration is performed either to any given plateau maximum rate or to a SPE fraction level measured in the field in real time.

During the period that preceded the implementation of this calibration procedure, the UC was deployed and operated to validate the muon detector design. The voltage at which the deployed SMs were operated was $960 \mathrm{~V}$ and with a uniform discrimination level of $100 \mathrm{mV}$ set for every channel of every module. We performed a calibration run at $960 \mathrm{~V}$ to evaluate how these fixed parameters (high voltage and threshold level) are reflected in the quality of data. There is a counting efficiency loss as thresholds move away from the plateaus and this loss occurs more rapidly in the $10 \mathrm{~m}^{2}$ modules. The loss of efficiency arises from the fact that as we inspect SPEs with higher thresholds some are rejected since either they fall short of the threshold of the digital discriminator or the digital signal is too short for the $3.125 \mathrm{~ns}$ sampling period. So both types of modules are in principle affected by this efficiency loss, but the loss is more pronounced for the $10 \mathrm{~m}^{2}$ module due to the reduced number of SPEs coming from the far end [10]. Also the plateau regions become narrower with lower PMT bias voltages, more evidently so for the $10 \mathrm{~m}^{2}$ modules. A uniform $100 \mathrm{mV}$ threshold was found to be essentially working for the $5 \mathrm{~m}^{2}$ modules in the data acquisition period mentioned above, shown in Fig. 2 (right panel). Therefore we will restrict the analysis presented in this work only to these modules. The complete data set considered in this work comprises all 
the cosmic ray events recorded during the first 20 months of the UC operation from March 2013, during the period previous to the implementation of the calibration routines. In this period six $5 \mathrm{~m}^{2}$ modules were operational: four in KT and two in PC. The UC has modules of two different sizes to study the counting efficiency and the saturation range with respect to the shower core position. AMIGA is designed to work with $30 \mathrm{~m}^{2}$ MCs divided in three $10 \mathrm{~m}^{2}$ modules. We combined the information of $2 \times 5 \mathrm{~m}^{2}$ modules for each event, to analyse the muon counts for $10 \mathrm{~m}^{2}$ of detection area. We added muon counts from the $5 \mathrm{~m}^{2} \mathrm{SMs}$ at the KT South twin position, at the KT North twin, and at the PC South twin. In this way we performed our analyses over three independent $10 \mathrm{~m}^{2}$ detection areas. The infill stations provide the geometry and energy reconstruction of the cosmic showers, 1235 events with zenith angle up to $45^{\circ}$, with energies higher than $10^{17} \mathrm{eV}$. Stations at least at $200 \mathrm{~m}$ away from the shower core were considered. The reconstruction algorithm for the events triggering the infill array is based on the code for the regular surface detector array. After selecting the signals which are generated by air showers, the direction and the energy of the primary cosmic ray are deduced from the timing information and from the total recorded signal in the stations [1].

\section{Detector Resolution}

As done to study the accuracy of the SD stations [11], the twin detectors can be used to study the fluctuations of the measured signals and preliminary results for the MC counting accuracy have been reported [10]. Having two detectors measuring basically the same spot on the shower allows us to estimate the signal fluctuation by analysing the difference of their signals for a given event. Considering that the separation between twin detectors is $\sim 20 \mathrm{~m}$, only AMIGA signals at least $200 \mathrm{~m}$ away from the shower axis are considered. In this way we can assume that both detectors are measuring samples coming from the same muon density.

The linear correlation between KT-North and KT-South is shown in Fig. 3 (left panel), where only events with at least 1 muon over a $10 \mathrm{~m}^{2}$ area are considered. From the slope of the linear fit of $1.05 \pm 0.02$, we can consider that the detectors respond in the same way for each event, a hypothesis needed to determine the resolution based on these data.

For each event we construct the resolution estimator $\Delta$ based on the sample variance and mean, define as follows: $\Delta^{2}=(\sigma / \bar{N})^{2}$, where $\sigma^{2}$ and $\bar{N}$ are the variance and mean estimators respectively calculated from the number of measured muons by each twin.

We extract the mean value of $\Delta^{2}$ within bins of average number of muons. In Fig. 3 (right panel), the values obtained from each $\Delta^{2}$ bin are displayed as a function of the muon count. For an ideal Poisson counter, the resolution should be $\Delta_{\text {poisson }}^{2}=N_{\mu}^{-1}$. The blue line is the fit of our model to the data. The muon detector resembles the ideal behaviour in this range of signals. From this analysis we can establish the counting uncertainty as a function of the number of counted muons as $\Delta^{2}\left(N_{\mu}\right)=(0.8 \pm 0.2) / N_{\mu}^{(0.9 \pm 0.1)}$.

\section{Muon Lateral Distribution}

For studying the lateral distribution of muons we choose a KASCADE-Grande-like LDF [8], since we are also counting muons over $10 \mathrm{~m}^{2}$ areas and the energy range of both experiments 

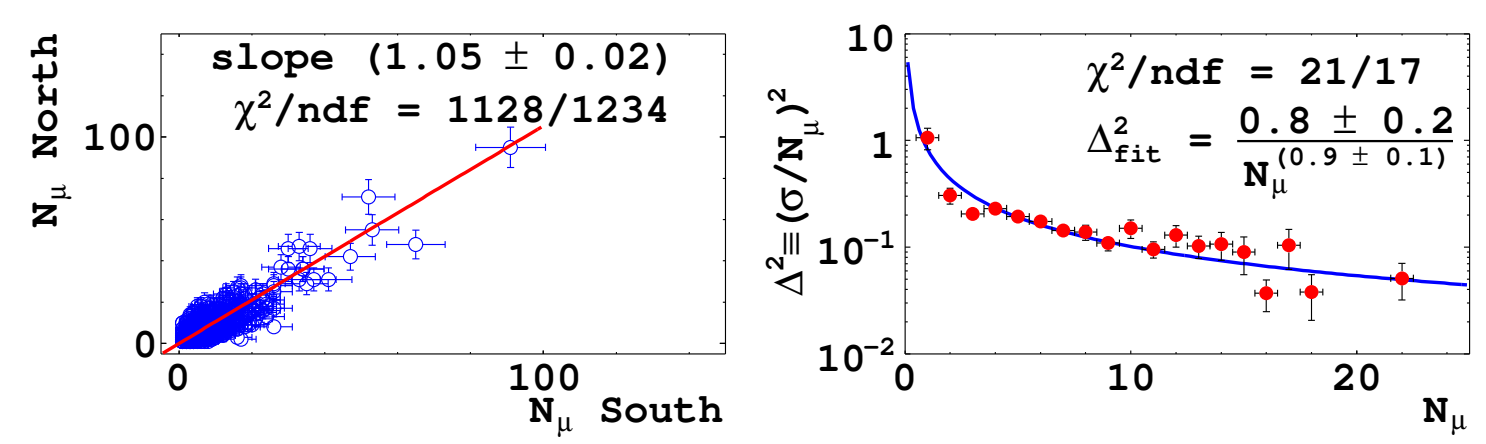

Figure 3: (Left) Muon counting comparison between twin counters at KT for events with $\theta \leq 45^{\circ}$, $\log (E / \mathrm{eV}) \geq 17.0$, at least $200 \mathrm{~m}$ from the shower core, and at least 1 muon over the $10 \mathrm{~m}^{2}$ area considered. (Right) Parametrisation of the muon counting resolution. The fitted model is displayed by the blue line.

overlaps. This is given by

$$
\begin{aligned}
N_{\mu}(r, E) & =N_{0}(E) \cdot f_{\mu}(r) / f_{\mu}\left(r_{0}\right) \\
f_{\mu}(x) & =\left(\frac{x}{r^{\star}}\right)^{-\alpha}\left(1+\frac{x}{r^{\star}}\right)^{-\beta}\left(1+\left(\frac{x}{10 \cdot r^{\star}}\right)^{2}\right)^{-\gamma}
\end{aligned}
$$

where $\alpha=1, \gamma=1.85$ and $r^{\star}=150 \mathrm{~m}$ are fixed parameters from Monte Carlo simulations. $N_{0}(E)=N_{\mu}\left(r_{0}=450 \mathrm{~m}, E\right)$ is the number of muons at the optimal distance from the shower axis. At $r_{0}$, the fluctuations of the LDF fit are minimised, this distance being mostly determined by the spacing between the detectors [12]. We analyse the data set for getting an effective value for the slope $\beta$ instead of using a simulation-driven result, leaving only the composition-sensitive parameter $N_{0}$ to be fitted for each event.

The method of finding an effective $\beta$ has two iterations. Firstly, we determine $N_{0}(E)$, i.e. we study the relation between the number of muons at the optimal distance and the energy. Secondly, we use this relation to normalise each event and, in this way, we are able to fit $f_{\mu}(r)$ of eq. 5.1 to all the events together and get an effective value of $\beta$ as a result.

The relation between $N_{0}$ and $E$ is derived from a subset of events for which the highest SD signal is found within the UC. We defined a Lateral Trigger Probability (LTP) for the muon detector, and demand LTP $\geq 90 \%$ at $450 \mathrm{~m}$ from the shower core, which implies an energy cut of $E>10^{17.3} \mathrm{eV}$. Above this energy threshold the trigger efficiency of the infill is $\geq 90 \%$. We define the LTP at different core distances in the range of 200-1000 m. Trigger probabilities are also obtained within energy bins. For a fixed energy range, we calculate in distance range the ratio of the number of muon counters with at least 1 count to the total number of muon counters that received an SD trigger. Uncertainties in the trigger probability are given by the binomial error. As already mentioned, we are only considering three independent $10 \mathrm{~m}^{2}$ muon-detection areas as muon counters in this work (see Sec. 3). In Fig. 4 (left panel) the muon trigger probability is shown for several energy ranges as a function of the distance to the shower axis. 

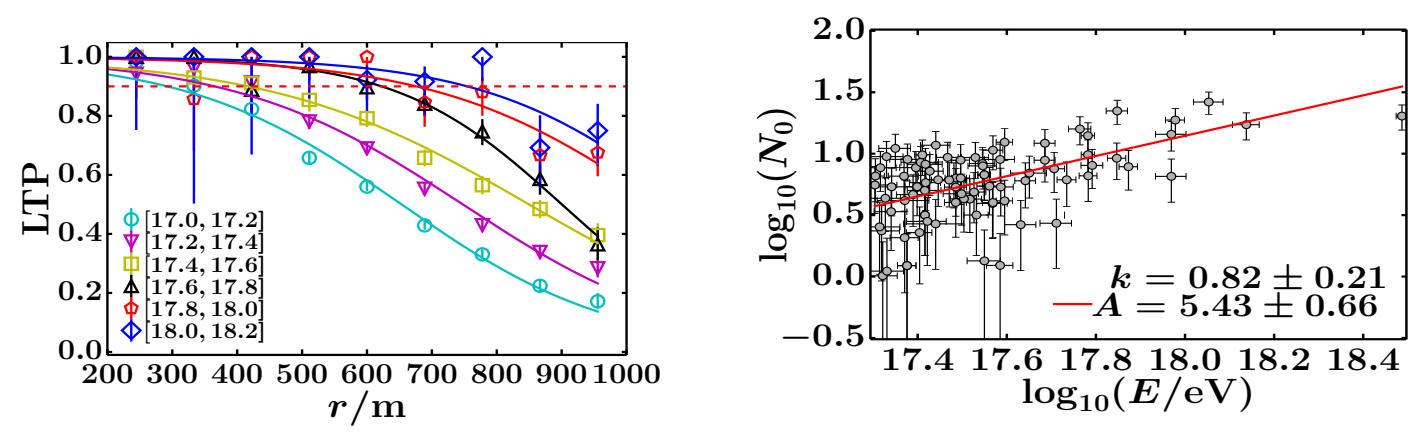

Figure 4: (Left) Muon counter lateral trigger probability as a function of distance from the shower core, for different energy ranges in $\log (E / \mathrm{eV})$. (Right) Determination of $N_{0}(E)$ with 100 selected events from muon counters within a distance $(450 \pm 25) \mathrm{m}$ from the shower core, and trigger probability higher than $90 \%$.
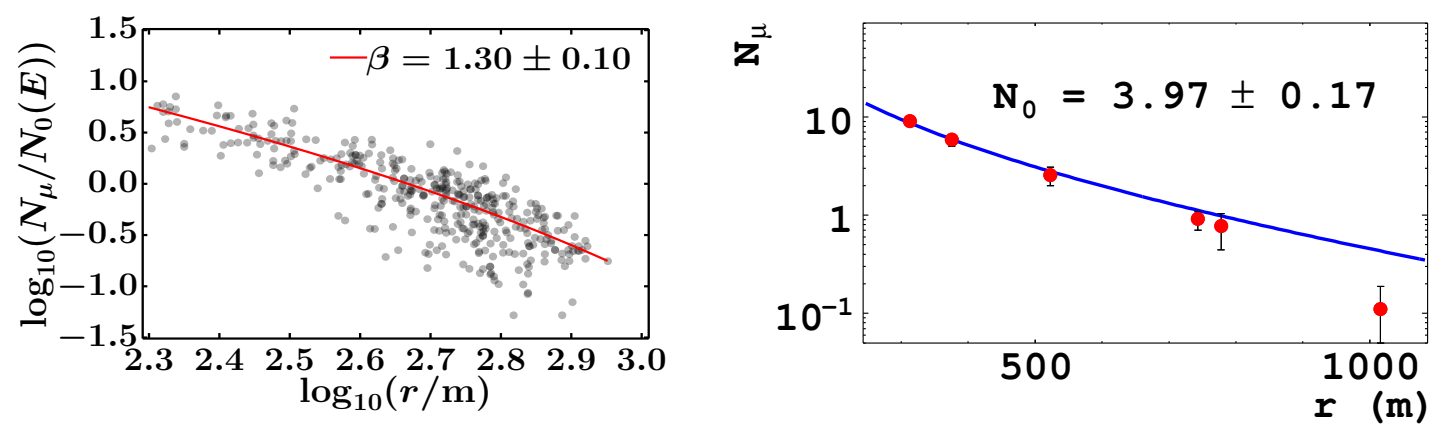

Figure 5: (Left) Muon lateral distribution for normalised data; an effective value for the slope $\beta$ is found and can be use for individual event fits. (Right) As a preliminary result, an LDF fit fixing $\beta$ is shown for a $10^{18.3} \mathrm{eV}$ event, where only $N_{0}$ remains as a free parameter.

The events shown in Fig. 4 (right panel) are fitted with the function,

$$
N_{0}(E)=A \cdot\left(\frac{E}{10^{17.5} \mathrm{eV}}\right)^{k}
$$

where $A=(5.43 \pm 0.66) \mathrm{m}^{-2}$ and $k=0.82 \pm 0.21$. A general maximum-likelihood approach developed in [13] is maximised to obtain the parameters. It is encouraging to note that the value of the scaling exponent $k$ is in agreement within statistical uncertainties with the one found by Akeno for the conversion between the number of muons and the energy [4].

To determine an effective value for $\beta$ we take a subset of events above the infill full efficiency, i.e. $10^{17.5} \mathrm{eV}$, and those muon detectors within a core distance for which their LTP $\geq 90 \%$. Each of the 389 inputs of this subset is normalised to $N_{0}(E)$ of eq. 5.2 thus eliminating the energy dependence. In Fig. 5 (left panel) the normalised number of muons is shown as a function to the shower axis. The subset is fitted with $f_{\mu}(r)$ of eq. 5.1 leading to an effective value of $\beta=1.3 \pm 0.1$ to be used for individual events. In Fig. 5 (right panel) we show an example of the application of this value of $\beta$ on a single event fitting. The event has $E=10^{18.3} \mathrm{eV}$, and only $N_{0}$ remains as a free parameter. 


\section{Summary}

The Unitary Cell of AMIGA has been completed since February 2015. It includes 37 scintillator modules in a hexagonal layout. In this work, we analysed the muon data over the first 20 months of operation.

We showed a method for finding a parametrisation of the muon counting resolution, to be used on an event by event basis, i.e. $\Delta^{2}\left(N_{\mu}\right)=(0.8 \pm 0.2) / N_{\mu}^{(0.9 \pm 0.1)}$. The scintillation module response resembles that of a Poisson counter.

Estimating the muon lateral trigger probability, we selected a fair sample for the parametrisation of the number of muons over $10 \mathrm{~m}^{2}$ at the optimal distance for the shower axis, as a function of the energy of the primary cosmic ray particle. This relation allows us to normalise the muon data in order to obtain an LDF fit performed on an energy-independent data set. This method led us to a value for the slope $\beta=1.3 \pm 0.1$ to be used for single event fitting.

Future work is needed that includes a more detailed comparison with results from previous experiments cited in the introduction. Such analysis will have to deal with the different conditions in which these results were found: different muon energy threshold, different lateral distribution and optimal distances, and different shielding.

\section{References}

[1] The Pierre Auger Collaboration accepted for publication in Nucl. Instrum. Meth. A (2015) [arXiv:1502.1323].

[2] R. Engel for the Pierre Auger Collaboration Proc. 34th ICRC 686 (2015).

[3] M. A. Lawrence et al. J. Phys. G 17 (1991) 733-757.

[4] The Akeno Collaboration J. Phys. G 21 (1995) 1101-1119.

[5] S. P. Knurenko et al. Int. J. Mod. Phys. A 20 (2005) 6900-6902.

[6] The AGASA Collaboration Nucl. Instrum. Meth. A 311 (1992) 338-349.

[7] The KASCADE Collaboration Nucl. Instrum. Meth. A 513 (2003) 490-510.

[8] The KASCADE-Grande Collaboration Nucl. Instrum. Meth. A 620 (2010) 202-216.

[9] B. Wundheiler for the Pierre Auger Collaboration Proc. 32nd ICRC 341 (2011) [arXiv:1107.4807].

[10] S. Maldera for the Pierre Auger Collaboration Proc. 33rd ICRC 748 (2013) [arXiv: 1307. 5059 ].

[11] M. Ave et al. Nucl. Instrum. Meth. A 578 (2007) 180-184.

[12] D. Ravignani et al. Astropart. Phys. 65 (2015) 1-10.

[13] H. P. Dembinski et al. submitted to Astropart. Phys. (2015) [arXiv:1503.9027]. 